\title{
Highly Chemo- and Regioselective Intermolecular Cyclotrimerization of Alkynes Catalyzed by Cationic Rhodium(I)/Modified BINAP Complexes
}

\author{
Ken Tanaka* and Kaori Shirasaka \\ Department of Applied Chemistry, Tokyo University of Agriculture and Technology, \\ Koganei, Tokyo 184-8588, Japan
}

\section{General}

Anhydrous $\mathrm{CH}_{2} \mathrm{Cl}_{2}$ was obtained from Aldrich (No. 27,099-7) and used as received. TolBINAP, H8-BINAP, and DTBM-SEGPHOS were obtained from Takasago International Corp. All other reagents were obtained from commercial sources and used as received. All reactions were carried out under an atmosphere of argon in oven-dried glassware with magnetic stirring, unless otherwise indicated.

\section{Cyclotrimerization of Terminal Alkynes (eq 2)}

General Procedure. Under a Ar atmosphere, DTBM-SEGPHOS (59.0 mg, $0.050 \mathrm{mmol}$ ) and $\left[\mathrm{Rh}(\mathrm{cod})_{2}\right] \mathrm{BF}_{4}(20.3 \mathrm{mg}, 0.050 \mathrm{mmol})$ were dissolved in $\mathrm{CH}_{2} \mathrm{Cl}_{2}(1.0 \mathrm{~mL})$ and the mixture was stirred for 5 minutes. $\mathrm{H}_{2}$ was introduced to the resulting solution in Schlenk tube. After stirring for 0.5 hour at room temperature, the resulting solution was concentrated to dryness. 1Dodecyne (1a) (166 mg, $1.0 \mathrm{mmol}$ ) was added to the residue by using $\mathrm{CH}_{2} \mathrm{Cl}_{2}(2.0 \mathrm{~mL})$. The mixture was stirred at room temperature for 24 hours. The resulting solution was concentrated and purified by preparative TLC (hexane), which furnished a mixture of 1,2,4-tridecylbenzene (2a) and 1,3,5-tridecylbenzene (3a) (152 mg, $0.913 \mathrm{mmol}, 91 \%, \mathbf{2 a : 3 a}=83: 17)$.

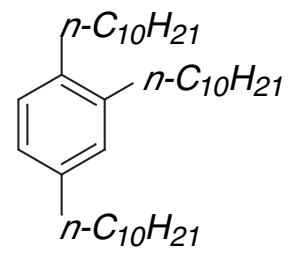

$2 a$

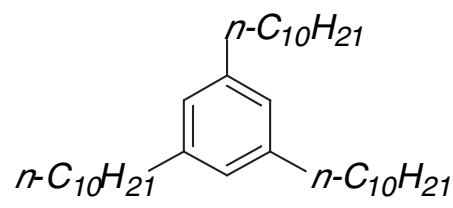

$3 a$

1,2,4-Tridecylbenzene (2a, 83\% regioselectivity). ${ }^{1}$ Colorless oil; ${ }^{1} \mathrm{H} \mathrm{NMR}\left(\mathrm{CDCl}_{3}, 300 \mathrm{MHz}\right)$ $\delta 7.04(\mathrm{~d}, J=7.5 \mathrm{~Hz}, 1 \mathrm{H}), 6.94(\mathrm{~s}, 1 \mathrm{H}), 6.92(\mathrm{~d}, J=7.5 \mathrm{~Hz}, 1 \mathrm{H}), 2.48-2.61(\mathrm{~m}, 6 \mathrm{H}), 1.45-1.65$ 
$(\mathrm{m}, 6 \mathrm{H}), 1.20-1.43(\mathrm{~m}, 42 \mathrm{H}), 0.88(\mathrm{t}, J=6.6 \mathrm{~Hz}, 9 \mathrm{H})$; aryl protons of minor 3a: $\delta 6.80(\mathrm{~s}, 3 \mathrm{H})$;

${ }^{13} \mathrm{C} \mathrm{NMR}\left(\mathrm{CDCl}_{3}, 75 \mathrm{MHz}\right) \delta 142.7,140.3,140.2,137.7,129.2,128.9,125.8,125.7,36.0,35.6$, $32.8,32.4,31.9,31.63,31.62,31.4,29.9,29.7,29.65,29.59,29.56,29.49,29.38,22.7,14.1$.

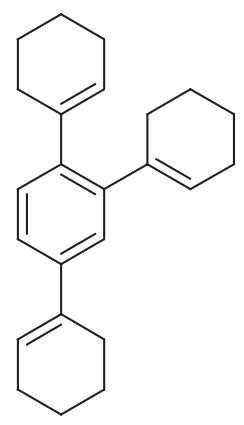

2b

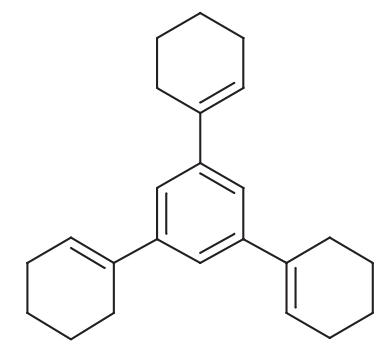

$3 b$

1,2,4-Tri-1-cyclohexenylbenzene $\left(2 \mathrm{~b}, 97 \%\right.$ regioselectivity). ${ }^{2}$ Yellow oil; ${ }^{1} \mathrm{H}$ NMR $\left(\mathrm{CDCl}_{3}, 300 \mathrm{MHz}\right) \delta 7.18(\mathrm{dd}, J=8.1$ and $2.1 \mathrm{~Hz}, 1 \mathrm{H}), 7.13(\mathrm{~d}, J=2.1 \mathrm{~Hz}, 1 \mathrm{H}), 7.04(\mathrm{~d}, J=8.1$ $\mathrm{Hz}, 1 \mathrm{H}), 6.06-6.14(\mathrm{~m}, 1 \mathrm{H}), 5.64-5.72(\mathrm{~m}, 2 \mathrm{H}), 2.04-2.48(\mathrm{~m}, 12 \mathrm{H}), 1.51-1.82$ (m, 12H); aryl

protons of minor 3b: $\delta 7.23(\mathrm{~s}, 3 \mathrm{H}) ;{ }^{13} \mathrm{C} \mathrm{NMR}\left(\mathrm{CDCl}_{3}, 75 \mathrm{MHz}\right) \delta 142.5,141.0,140.6,139.8$, $139.1,136.3,128.4,126.2,125.3,124.1,123.4,122.9,29.59,29.54,29.48,27.4,25.88,25.77$, $25.76,25.73,23.3,23.1,22.2$.

\section{Cocyclotrimerization of Diethyl Acetylenedicarboxylate and Terminal Alkynes} (Table 2)

General Procedure (entry 1). Under a Ar atmosphere, H8-BINAP (5.7 mg, 0.009 mmol) and $\left[\mathrm{Rh}(\operatorname{cod})_{2}\right] \mathrm{BF}_{4}(3.7 \mathrm{mg}, 0.009 \mathrm{mmol})$ were dissolved in $\mathrm{CH}_{2} \mathrm{Cl}_{2}(1.0 \mathrm{~mL})$ and the mixture was stirred for 5 minutes. $\mathrm{H}_{2}$ was introduced to the resulting solution in Schlenk tube. After stirring for 0.5 hour at room temperature, the resulting solution was concentrated to dryness and dissolved in $\mathrm{CH}_{2} \mathrm{Cl}_{2}(2.0 \mathrm{~mL})$. To this solution was added dropwise over 1 minute a solution of 1-dodecyne (1a) $(99.8 \mathrm{mg}, 0.60 \mathrm{mmol})$ and diethyl acetylenedicarboxylate (4) (51.0 mg, 0.30 $\mathrm{mmol})$ in $\mathrm{CH}_{2} \mathrm{Cl}_{2}(0.5 \mathrm{~mL})$ and the solution was stirred at room temperature for 1 hour. The resulting solution was concentrated and purified by preparative TLC (hexane:ethyl acetate $=$ 10:1), which furnished a mixture of 3,6-diphenylphthalic acid diethyl ester (5a), 3,5didecylphthalic acid diethyl ester (6a), and 4,5-didecylphthalic acid diethyl ester (7a) (133 mg, 
$0.264 \mathrm{mmol}, 88 \%$, 5a:6a:7a $=92: 6: 2)$.

This mixture could be purified by preparative TLC (hexane:ethyl acetate $=10: 1$ ), which furnished pure 3,6-diphenylphthalic acid diethyl ester (5a) (120 mg, $0.238 \mathrm{mmol}, 79 \%$ ).

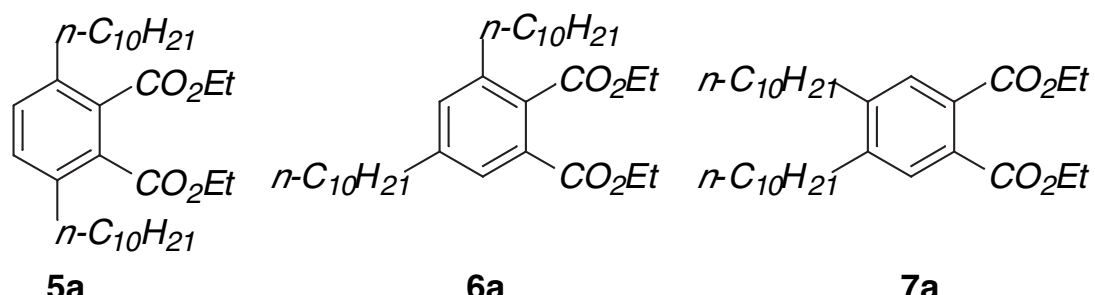

3,6-Didecylphthalic acid diethyl ester (5a, entry 1). Colorless solid; Mp $44{ }^{\circ} \mathrm{C}$ (pure 5a); IR (neat), 2750, 1670, 1230, 1160, $1060 \mathrm{~cm}^{-1} ;{ }^{1} \mathrm{H}$ NMR $\left(\mathrm{CDCl}_{3}, 300 \mathrm{MHz}\right) \delta 7.20$ (s, 2H), 4.32 (q, $J$ $=7.2 \mathrm{~Hz}, 4 \mathrm{H}), 2.67(\mathrm{t}, J=7.8 \mathrm{~Hz}, 4 \mathrm{H}), 1.50-1.62(\mathrm{~m}, 4 \mathrm{H}), 1.36(\mathrm{t}, J=7.2 \mathrm{~Hz}, 6 \mathrm{H}), 1.18-1.40(\mathrm{~m}$, $28 \mathrm{H}), 0.88(\mathrm{t}, J=6.6 \mathrm{~Hz}, 6 \mathrm{H})$; aryl protons of minor $6 \mathbf{a}: \delta 7.62(\mathrm{~d}, J=1.8 \mathrm{~Hz}, 1 \mathrm{H}), 7.20(\mathrm{~d}, J=$ $1.8 \mathrm{~Hz}, 1 \mathrm{H})$; aryl protons of minor $7 \mathbf{a}: \delta 7.48(\mathrm{~s}, 2 \mathrm{H}),{ }^{13} \mathrm{C} \mathrm{NMR}\left(\mathrm{CDCl}_{3}, 75 \mathrm{MHz}\right) \delta 168.6,139.0$, 131.8, 131.6, 61.3, 33.5, 31.9, 31.6, 29.6, 29.63, 29.60, 29.5, 29.3, 22.7, 14.12, 14.10. Anal. Calcd for $\mathrm{C}_{32} \mathrm{H}_{54} \mathrm{O}_{4}$ : C, 76.45; H, 10.83. Found: C, 76.73; H, 11.09.

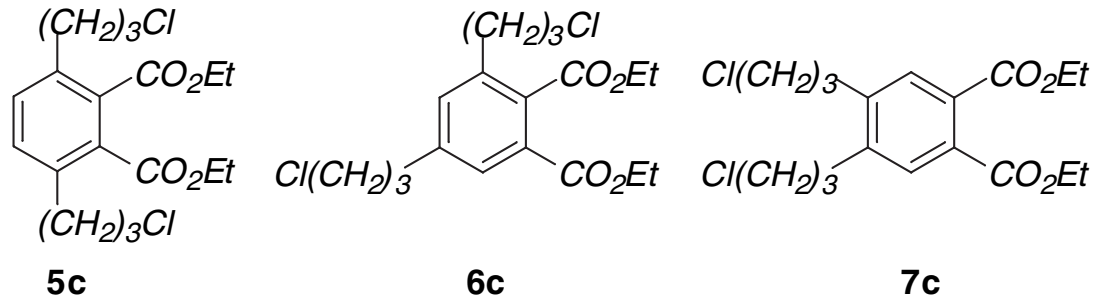

\section{3,6-Bis(3-chloropropyl)phthalic acid diethyl ester (5c, 91\% regioselectivity, entry 2).}

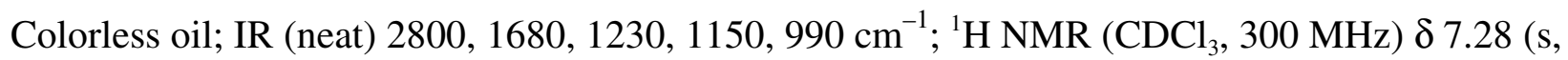
$2 \mathrm{H}), 4.34(\mathrm{q}, J=7.2 \mathrm{~Hz}, 4 \mathrm{H}), 3.54(\mathrm{t}, J=6.3 \mathrm{~Hz}, 4 \mathrm{H}), 2.86(\mathrm{t}, J=7.5 \mathrm{~Hz}, 4 \mathrm{H}), 2.02-2.11(\mathrm{~m}$, $4 \mathrm{H}), 1.37(\mathrm{t}, J=7.2 \mathrm{~Hz}, 6 \mathrm{H})$; aryl protons of minor $\mathbf{6 c}: \delta 7.68(\mathrm{~d}, J=1.5 \mathrm{~Hz}, 1 \mathrm{H}), 7.28(\mathrm{~d}, J=1.5$ $\mathrm{Hz}, 1 \mathrm{H})$; aryl protons of minor 7c: $\delta 7.53(\mathrm{~s}, 2 \mathrm{H}) ;{ }^{13} \mathrm{C} \mathrm{NMR}\left(\mathrm{CDCl}_{3}, 75 \mathrm{MHz}\right) \delta 168.1,137.6$, 132.4, 132.0, 61.7, 44.3, 34.0, 30.6, 14.1. Anal. Calcd for $\mathrm{C}_{18} \mathrm{H}_{24} \mathrm{O}_{4}$ : C, 57.61; $\mathrm{H}, 6.45$. Found: C, $57.89 ; \mathrm{H}, 6.51$. 


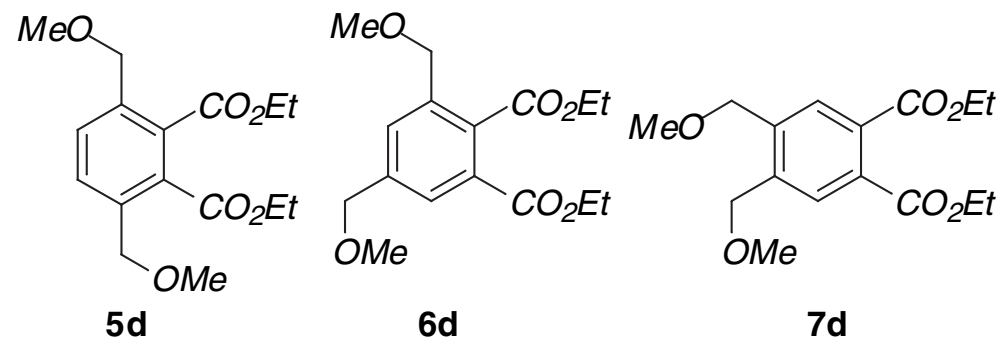

3,6-Bis(methoxymethyl)phthalic acid diethyl ester (5d, 86\% regioselectivity, entry 3). Colorless oil; IR (neat) 2750, 1660, 1220, 1070, $990 \mathrm{~cm}^{-1} ;{ }^{1} \mathrm{H}$ NMR $\left(\mathrm{CDCl}_{3}, 300 \mathrm{MHz}\right) \delta 7.54$ (s, 2H), $4.59(\mathrm{~s}, 4 \mathrm{H}), 4.33(\mathrm{q}, J=7.2 \mathrm{~Hz}, 4 \mathrm{H}), 3.35(\mathrm{~s}, 6 \mathrm{H}), 1.36(\mathrm{t}, J=7.2 \mathrm{~Hz}, 6 \mathrm{H})$; aryl protons of minor $\mathbf{6 d}: \delta$ 7.85-7.87 $(\mathrm{m}, 1 \mathrm{H}), 7.61-7.63(\mathrm{~m}, 1 \mathrm{H})$; aryl protons of minor $7 \mathbf{d}: \delta 7.77(\mathrm{~s}, 2 \mathrm{H}) ;{ }^{13} \mathrm{C}$ NMR $\left(\mathrm{CDCl}_{3}, 75 \mathrm{MHz}\right) \delta 167.5,136.6,131.2,129.8,71.8,61.5,58.5,13.9$. Anal. Calcd for $\mathrm{C}_{16} \mathrm{H}_{22} \mathrm{O}_{4}: \mathrm{C}, 61.92 ; \mathrm{H}, 7.15$. Found C, 61.60; H, 6.95.

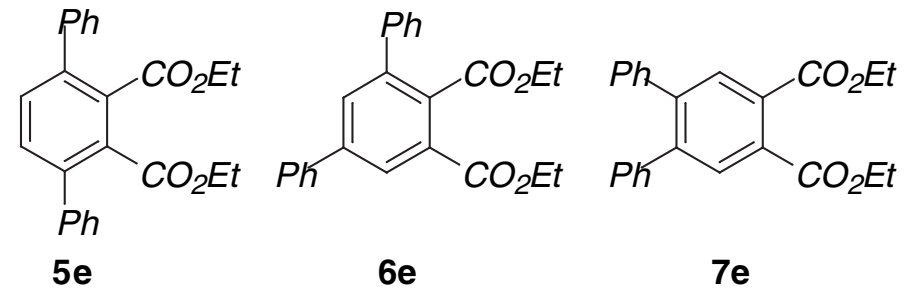

3,6-Diphenylphthalic acid diethyl ester (5e, $89 \%$ regioselectivity, entry 4). Colorless solid; Mp 99-105 ${ }^{\circ} \mathrm{C}$; IR (neat) 1680, 1200, 1110, 1040, 740, $670 \mathrm{~cm}^{-1} ;{ }^{1} \mathrm{H} \mathrm{NMR}\left(\mathrm{CDCl}_{3}, 300\right.$ MHz) $\delta 7.47$ (s, 2H), 7.32-7.66 (m, 10H), 4.07 (q, $J=7.2 \mathrm{~Hz}, 4 \mathrm{H}), 0.97(\mathrm{t}, J=6.9 \mathrm{~Hz}, 6 \mathrm{H})$; aryl protons of minor $6 \mathrm{e}: \delta 8.21(\mathrm{~d}, J=2.1 \mathrm{~Hz}, 1 \mathrm{H}), 7.74(\mathrm{~d}, J=2.1 \mathrm{~Hz}, 1 \mathrm{H})$; aryl protons of minor 7e: $\delta 7.79$ (s, 2H); ${ }^{13} \mathrm{C}$ NMR (CDCl $\left.3,75 \mathrm{MHz}\right) \delta 168.3,139.9,132.3,131.5,129.0,128.4,128.3$, 127.7, 61.5, 13.5. Anal. Calcd for $\mathrm{C}_{24} \mathrm{H}_{22} \mathrm{O}_{4}$ : C, 76.99; H, 5.92. Found: C, 76.82; H, 6.01.

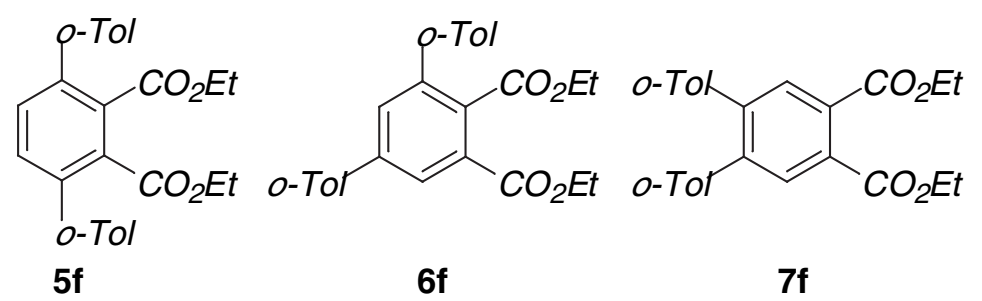

3,6-Di-o-tolylphthalic acid diethyl ester (5f, 89\% regioselectivity, entry 5). Colorless solid; $\mathrm{Mp} 103-108{ }^{\circ} \mathrm{C}$; IR (neat) 2800, 1680, 1380, 1200, 1100, $730 \mathrm{~cm}^{-1} ;{ }^{1} \mathrm{H}$ NMR $\left(\mathrm{CDCl}_{3}, 300 \mathrm{MHz}\right)$ $\delta 7.33(\mathrm{~s}, 2 \mathrm{H}), 7.07-7.29(\mathrm{~m}, 8 \mathrm{H}), 3.98(\mathrm{q}, J=7.2 \mathrm{~Hz}, 1.6 \mathrm{H}), 3.97(\mathrm{q}, J=7.2 \mathrm{~Hz}, 2.4 \mathrm{H}), 2.21$ (s, $3.5 \mathrm{H}), 2.17(\mathrm{~s}, 2.5 \mathrm{H}), 0.88(\mathrm{t}, J=7.2 \mathrm{~Hz}, 2.5 \mathrm{H}), 0.87(\mathrm{t}, J=7.2 \mathrm{~Hz}, 3.5 \mathrm{H})$; aryl protons of minor 
6f: $\delta 7.99(\mathrm{~d}, J=2.1 \mathrm{~Hz}, 1 \mathrm{H}), 7.38(\mathrm{~d}, J=2.1 \mathrm{~Hz}, 1 \mathrm{H})$; aryl protons of minor $7 \mathbf{f}: \delta 7.69(\mathrm{~s}, 2 \mathrm{H})$; ${ }^{13} \mathrm{C} \mathrm{NMR}\left(\mathrm{CDCl}_{3}, 75 \mathrm{MHz}\right) \delta 167.9,139.9,139.8,139.5,136.2,135.9,132.6,129.3,127.9$, 125.2, 61.2, 20.4, 13.4. Anal. Calcd for $\mathrm{C}_{26} \mathrm{H}_{26} \mathrm{O}_{4}:$ C, 77.59; H, 6.51. Found C, 77.41; H, 6.47.

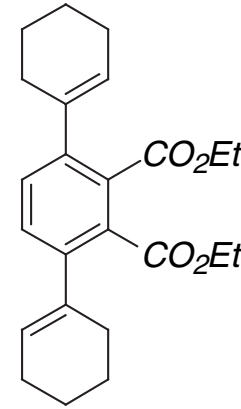

$5 b$

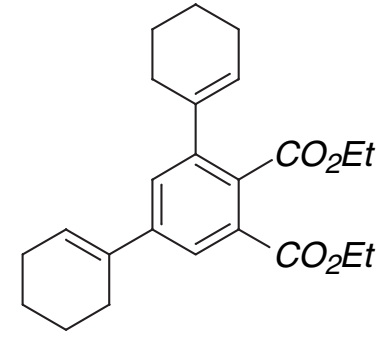

6b<smiles>CCOC(=O)c1cc(C2=CCCCC2)c(C2=CCCCC2)cc1C(=O)OCC</smiles>

$\mathbf{7 b}$

3,6-Di-1-cyclohexenylphthalic acid diethyl ester (5b, $91 \%$ regioselectivity, entry 6). Colorless oil; IR (neat) 3200, 2750 1660, 1180, $770 \mathrm{~cm}^{-1} ;{ }^{1} \mathrm{H}$ NMR $\left(\mathrm{CDCl}_{3}, 300 \mathrm{MHz}\right) \delta 7.18(\mathrm{~s}$, 2H), 5.57-5.62 (m, 2H), $4.26(\mathrm{q}, J=7.2 \mathrm{~Hz}, 4 \mathrm{H}), 2.03-2.33(\mathrm{~m}, 8 \mathrm{H}), 1.55-1.80(\mathrm{~m}, 8 \mathrm{H}), 1.33(\mathrm{t}, J$ $=7.2 \mathrm{~Hz}, 6 \mathrm{H})$; aryl protons of minor $6 \mathbf{b}: \delta 7.83(\mathrm{~d}, J=1.8 \mathrm{~Hz}, 1 \mathrm{H}), 7.33(\mathrm{~d}, J=1.8 \mathrm{~Hz}, 1 \mathrm{H})$; aryl protons of minor $7 \mathbf{b}: \delta 7.46(\mathrm{~s}, 2 \mathrm{H}) ;{ }^{13} \mathrm{C} \mathrm{NMR}\left(\mathrm{CDCl}_{3}, 75 \mathrm{MHz}\right) \delta 168.7,141.7,137.3,131.3$, 129.5, 126.7, 61.4, 29.8, 25.5, 23.1, 21.9, 14.1. Anal. Calcd for $\mathrm{C}_{24} \mathrm{H}_{30} \mathrm{O}_{4}$ : C, 75.36; H, 7.91. Found C, 74.87; H, 7.97.

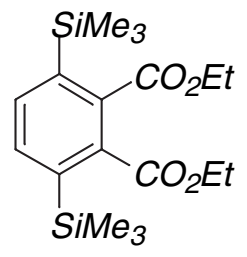

$5 \mathrm{~g}$

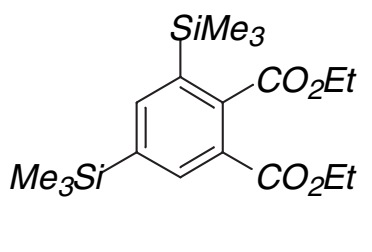

$6 \mathrm{~g}$

3,6-Bis(trimethylsilyl)phthalic acid diethyl ester (5g, 99\% regioselectivity, entry 7). Mp 65-68 ${ }^{\circ} \mathrm{C}$; IR (neat) 2770, 1660, 1200, 1130, 1070, $790 \mathrm{~cm}^{-1} ;{ }^{1} \mathrm{H}$ NMR $\left(\mathrm{CDCl}_{3}, 300 \mathrm{MHz}\right) \delta 7.65$ $(\mathrm{s}, 2 \mathrm{H}), 4.32(\mathrm{q}, J=7.2 \mathrm{~Hz}, 4 \mathrm{H}), 1.37(\mathrm{t}, J=7.2 \mathrm{~Hz}, 6 \mathrm{H}), 0.30(\mathrm{~s}, 18 \mathrm{H})$; aryl protons of minor

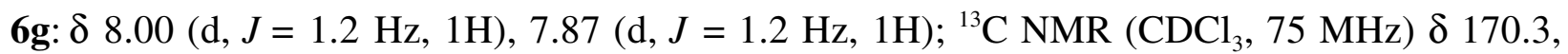
140.4, 137.7, 135.8, 61.9, 14.3, 0.3. Anal. Calad for $\mathrm{C}_{18} \mathrm{H}_{30} \mathrm{Si}_{2} \mathrm{O}_{4}: \mathrm{C}, 58.97 ; \mathrm{H}, 8.25$. Found C, $59.10 ; \mathrm{H}, 8.36$. 


\section{Synthesis of Metacyclophane}

Under a Ar atmosphere, H8-BINAP $(2.5 \mathrm{mg}, 0.00625 \mathrm{mmol})$ and $\left[\mathrm{Rh}(\mathrm{cod})_{2}\right] \mathrm{BF}_{4}(3.9 \mathrm{mg}$, $0.00625 \mathrm{mmol})$ were dissolved in $\mathrm{CH}_{2} \mathrm{Cl}_{2}(1.0 \mathrm{~mL})$ and the mixture was stirred for 5 minutes. $\mathrm{H}_{2}$ was introduced to the resulting solution in Schlenk tube. After stirring for 0.5 hour at room temperature, the resulting solution was concentrated to dryness and dissolved in $\mathrm{CH}_{2} \mathrm{Cl}_{2}$ (20 $\mathrm{mL})$. To this solution was added dropwise over 1 minute a solution of 1,9-decadiyne (8) (16.8 $\mathrm{mg}, 0.125 \mathrm{mmol})$ and diethyl acetylenedicarboxylate (4) $(21.3 \mathrm{mg}, 0.125 \mathrm{mmol})$ in $\mathrm{CH}_{2} \mathrm{Cl}_{2}(2.0$ $\mathrm{mL}$ ) and the solution was stirred at room temperature for 1 hour. The resulting solution was concentrated and purified by preparative TLC (hexane:ethyl acetate $=3: 1$ ), which furnished a metacyclophane 9 (19.0 mg, $0.0623 \mathrm{mmol}, 50 \%)$.

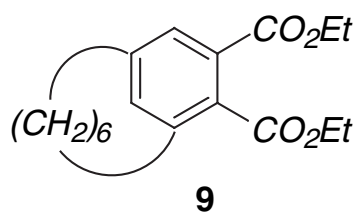

[6]Metacyclophane-8,9-dicarboxylic acid diethyl ester (9). Colorless oil; IR (neat) 2800, 1670, 1420, 1230, $750 \mathrm{~cm}^{-1}$; ${ }^{1} \mathrm{H} \mathrm{NMR}\left(\mathrm{CDCl}_{3}, 300 \mathrm{MHz}\right) \delta 7.50(\mathrm{~d}, J=1.2 \mathrm{~Hz}, 1 \mathrm{H}), 7.45(\mathrm{~d}, J=$ $1.2 \mathrm{~Hz}, 1 \mathrm{H}), 4.29-4.43$ (m, 4H), 2.54-2.84 (m, 4H), 1.72-1.91 (m, 2H), 1.16-1.48 (m, 4H), 1.36 $(\mathrm{t}, J=7.2 \mathrm{~Hz}, 6 \mathrm{H}), 0.20-0.65(\mathrm{~m}, 2 \mathrm{H}) ;{ }^{13} \mathrm{C} \mathrm{NMR}\left(\mathrm{CDCl}_{3}, 75 \mathrm{MHz}\right) \delta 168.9,166.7,144.2,141.1$, 139.3, 130.6, 129.5, 125.6, 61.3, 61.2, 34.3, 32.6, 32.4, 32.3, 27.8, 27.5, 14.13, 14.11. HRMS (FAB). Calad for $\mathrm{C}_{18} \mathrm{H}_{24} \mathrm{O}_{4}: 304.1753$. Found 304.1779. Anal. Calad for $\mathrm{C}_{18} \mathrm{H}_{24} \mathrm{O}_{4}: \mathrm{C}, 71.03 ; \mathrm{H}$, 7.95. Found C, 71.29; H, 7.96.

\section{References}

(1) Yong, L.; Butenschön, H. Chem. Commun. 2002, 23, 2852-2853.

(2) Dérien, S.; Clinet, J.-C.; Duñach, E.; Périchon, J. J. Organamet. Chem. 1992, 424, 213-224. 